\title{
The Anti-Inflammatory Effects of Phenolic Dental Medicaments as Determined by Mouse Ear Edema Assay
}

\author{
Toshihiro DOHI, Hiroshi TERADA ${ }^{1}$, Shinichi ANAMURA', \\ Hiroshi OKAMOTO ${ }^{1}$ and Akira TSUJIMOTO

\begin{abstract}
Department of Pharmacology and 'Department of Endodontology and Periodontology.
\end{abstract} \\ Hiroshima University School of Dentistry, 1-2-3 Kasumi, Minami-ku. Hiroshima 734, Japan
}

Accepted January 19,1989

\begin{abstract}
The anti-inflammatory effects of phenolic dental medicaments were evaluated by mouse ear edema assay. p-Chlorophenol (PCP) inhibited edema when applied topically in dosages of 0.2 and $0.5 \mathrm{mg}$ per site at $15 \mathrm{~min}$ before or 1.0 and $2.0 \mathrm{mg}$ per site at $60 \mathrm{~min}$ after the application of croton oil. The inhibitory effects were also noted with eugenol, guaiacol, o-cresol, phenol and orally administered indomethacin $(10 \mathrm{mg} / \mathrm{kg})$. The involvement of the effects on prostaglandin biosynthesis in the anti-inflammatory effects of these compounds is discussed.
\end{abstract}

The inhibition of prostaglandin (PG) synthesis by anti-inflammatory drugs has been demonstrated in a variety of cell types and tissues. It has become evident that the se!ective inhibition of cyclo-oxygenase by nonsteroidal anti-inflammatory drugs is the major mechanism of the anti-inflammatory action (1). Phenolic dental medicaments, widely used in root canal therapy as disinfectants, are also employed for the treatment of pulpitis and dentine hyperalgesia and produce an analgesic effect. The analgesic effect of phenolic dental medicaments has been believed to be due to their action as nonspecific counter-irritants, anesthesia dolorosa.

It has been demonstrated that phenol and its derivatives can inhibit PG biosynthesis in various preparations in vitro $(2,3)$. Various phenolic dental medicaments inhibit the release of $\mathrm{PGI}_{2}$ from rat dental pulp (4) and the conversion of arachidonic acid to $P G s$ by microsomes of bovine dental pulp (5). It has also been reported that essential oils (containing phenolic composites and used in the treatment of rheumatism and inflammation) interfered with PG biosynthesis. Besides the potent anti-bacterial and antifungal effects, phenolic medicaments may topically produce anti-inflammatory effects $v i a$ the inhibition of $P G$ biosynthesis.
However, the anti-inflammatory effects of phenolic dental medicaments are not wellknown. In addition, there are some anomalies between the inhibitory effects on cyclo-oxygenase in vitro and the biological effects in vivo. Furthermore, phenolic antioxidants serve as the reducing substrate of $P G$ hydroperoxidase and thus enhance the conversion of $P G G_{2}$ to $P G H_{2}$. They also scavenge hydroxyradicals which are produced during the breakdown of $P G_{2}$. Therefore phenolic antioxidants prevent PG synthase from inactivation by peroxide and radicals. We have shown that some phenolic medicaments such as p-chlorophenol (PCP) stimulated PG biosynthesis by microsomes of bovine dental pulp and rabbit renal medulla at low concentrations, while they inhibited it at high concentrations, and other agents such as eugenol were solely inhibitory (5). The present study examined the anti-inflammatory activity of phenolic dental medicaments by the mouse ear edema assay. The phenolic medicaments which have the following different effects on PG synthesis (5) were chosen: both stimulatory and inhibitory effects depending on the concentrations (phenol, PCP and o-cresol), small stimulatory and potent inhibitory effects (guaiacol) and solely inhibitory effects (eugenol).

Male dd mice weighing about $20 \mathrm{~g}$ were 
used. Anti-inflammatory effects were evaluated by the mouse ear edema assay described by Van Arman (6). In brief, $50 \mu$ l of solution containing $2 \%$ croton oil, $20 \%$ pyridine, $73 \%$ diethyl ether and $5 \%$ distilled water was applied to each site on the surface of the right ear under ether anesthesia. The left ear was left untreated. The drugs to be tested were dissolved in a vehicle containing $75 \%$ diethyl ether, $20 \%$ pyridine and $5 \%$ water. The solution ( $50 \mathrm{kl}$ each) was applied on both surfaces of the right ear at $15 \mathrm{~min}$ prior to or 1 hour after the application of croton oil. Only the vehicle was applied for the control. Four hours after the application of croton oil. the mice were sacrificed by cervical dislocation, and circular sections $(7 \mathrm{~mm}$ diameter) were taken from both ears using a tissue isolation punch. The increase in weight caused by croton oil was obtained by subtracting the weight of the untreated left ear section from that of the right ear section. The values in the results represent the percent increase in weight of the croton oil-treated ears compared with the untreated ears. Ten to 20 mice per group were used.

Pretreatment of PCP, $0.2-0.5 \mathrm{mg}$ per site, significantly inhibited croton oil-induced increases in tissue weight (Fig. 1A). The inhibitory effect of PCP, however, decreased in higher dosages. This may be due to an irritation effect at high dosages of PCP. which acts synergistically with croton oil. However, PCP had no significant effect on ear weight by itself. Significant inhibition was also observed when 1-2 $\mathrm{mg}$ of PCP was applied after the treatment with croton oil (Fig. 1B). Thus, the inhibitory effect of PCP was not an apparent anti-edematous effect resulting from denaturation of ear surface protein by PCP. which would prevent components of croton oil from penetrating and acting. The effects of other phenolic medicaments on croton oil-induced edema formation were also examined (Table 1). Eugenol, guaiacol and o-cresol produced a significant inhibitory effect within a range of dosage similar to that used for PCP, but phenol required somewhat higher dosages. Orally administered indomethacin also reduced the formation of edema.

A phenolic compound. MK 447 (2aminomethyl-4-t-6-iodophenol), is an effective anti-inflammatory agent in vivo, even though it stimulates PG biosynthesis (7). Similar results were also reported with phenol (7). In recent vears, evidence has been presented that oxygen free-radicals have an important role in inflammatory diseases. It
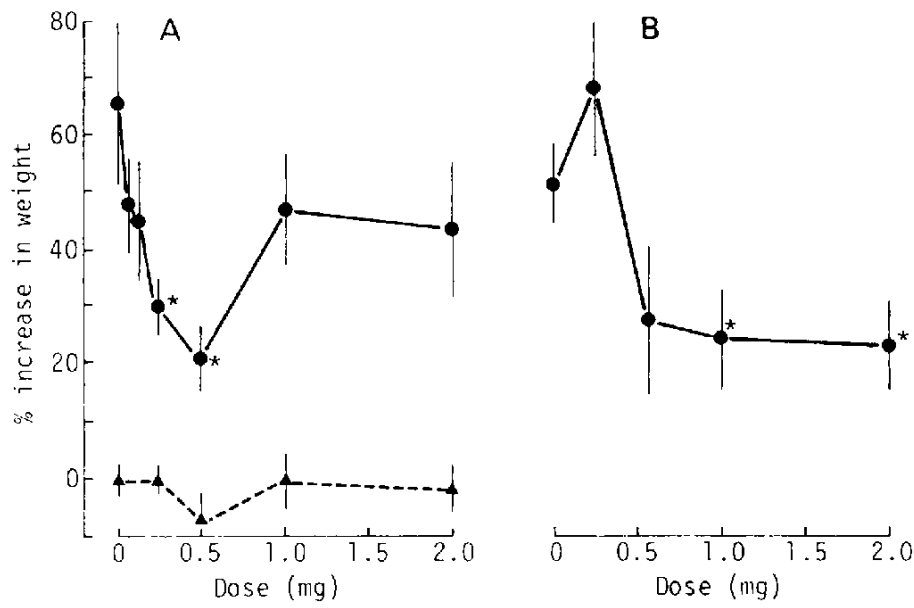

Fig. 1. Effects of p-chlorophenol (PCP) on mouse ear edema induced by croton oil. PCP was applied to each site of the ear at $15 \mathrm{~min}$ before $(A)$ and $60 \mathrm{~min}$ after (B) the application of croton oil. Dosages given are the dosages applied to each site. The solid line represents the percent of increase in weight of the croton oil-treated ear compared with the untreated ear. The dotted line represents the changes in weight of the ear caused by PCP in the absence of croton oil. "Significantly different from the control by Student's $t$-test, $P<0.05$. 
Table 1. Effects of phenolic compounds on the edema of mouse ear induced by croton oil

\begin{tabular}{|c|c|c|c|}
\hline Drugs & .... & $\begin{array}{c}\text { Dose } \\
\mathrm{mg}\end{array}$ & $\begin{array}{c}\% \text { increase in } \\
\text { weight }\end{array}$ \\
\hline \multicolumn{4}{|l|}{ Phenol } \\
\hline & & 0 & $71.9 \pm 13.2$ \\
\hline & & 0.2 & $59.7 \pm 10.6$ \\
\hline & & 0.5 & $54.2 \pm 13.1^{*}$ \\
\hline & & 1.0 & $40.5 \pm 8.5^{*}$ \\
\hline & & 2.0 & $64.3 \pm 11.1$ \\
\hline \multicolumn{4}{|l|}{ Eugenol } \\
\hline & & 0 & $76.1 \pm 14.8$ \\
\hline & & 0.1 & $51.6 \pm 12.1$ \\
\hline & & 0.2 & $34.1 \pm 5.7^{*}$ \\
\hline & & 0.5 & $39.8 \pm 8.4^{*}$ \\
\hline & & 1.0 & $56.5 \pm 11.2$ \\
\hline \multicolumn{4}{|l|}{ Guaiacol } \\
\hline & & 0 & $30.0 \pm 5.6$ \\
\hline & & 0.1 & $19.2 \pm 8.0$ \\
\hline & & 0.2 & $13.5 \pm 5.1^{*}$ \\
\hline & & 0.5 & $23.6 \pm 9.1$ \\
\hline & & 1.0 & $39.5 \pm 14.2$ \\
\hline \multicolumn{4}{|l|}{ o-Cresol } \\
\hline & & 0 & $63.3 \pm 10.3$ \\
\hline & & 0.1 & $43.1 \pm 11.1$ \\
\hline & & 0.2 & $33.8 \pm 6.6^{*}$ \\
\hline & & 0.5 & $23.6 \pm 9.1^{*}$ \\
\hline & & 1.0 & $85.8 \pm 12.0$ \\
\hline (Indomethacin) & & 10 & $29.2 \pm 7.5^{*}$ \\
\hline
\end{tabular}

Drugs were applied to each site of the ear at $15 \mathrm{~min}$ before the application of croton oil. Indomethacin. $10 \mathrm{mg} / \mathrm{kg}$. Was orally administered at $1 \mathrm{hr}$ before the application of croton oil. "Significantly different from the control by Student's $t$-test. $P<0.05$.

has been suggested that the anti-inflammatory activity of some drugs may be partly due to their ability to interfere with radical-mediated reactions. Azuma et al. (8) reported that phenolic compounds inhibited neutrophil chemotaxis. They suggested that the prevention of the production of oxygen free-radicals by inhibiting leukocyte chemotaxis with phenolic compounds is one mechanism of the anti-inflammatory action of such compounds. Kuehi et al. (7) suggested that $\mathrm{PGG}_{2}$ and $\mathrm{OH}$ which is generated during conversion of $P G G_{2}$ to $P G H_{2}$ are the important mediators of inflammation rather than $\mathrm{PGE}_{2}$ and $P G F_{2 \alpha}$. They also proposed that the antiinflammatory actions of MK 447 and phenol are accomplished by diminishing the amount of $P G G_{2}$ by stimulating the conversion of $P G G_{2}$ to $P \mathrm{PH}_{2}$ and by scavenging the radicals. However, the present results showed that the anti-inflammatory effects of phenolic compounds were not correlated with the stimulatory effect on PG biosynthesis because eugenol and guaiacol which had little or no stimulatory action on PG biosynthesis produced the effective anti-inflammatory effect as evaluated by the mouse ear edema assay. Dissociation between the anti-inflammatory action of phenolic anti-inflammatory drugs and their activities as radical scavengers or stimulators of $P G$ biosynthesis has also been reproted. For example, only the (+)isomer of MK 447 was biologically active, although both the $(+)$ and $(-)$ isomers were equipotent radical scavengers and stimulators of $P G$ biosynthesis (9). In addition. MK 447 analogues are equally potent as antioxidants but differentially active as an inhibitor of 
cyclooxygenase: the $(+)$ isomer inhibited, but the (-)isomer did not (10). The inhibitory action of MK 447 on PG biosynthesis under some conditions have recently been reported. Harada et al. (11) reported that low concentrations of MK 447 stimulated and high concentrations inhibited $P G$ endoperoxide formation from arachidonic acid by bovine seminal vesicle microsomes. Lands (12) suggested that a smal! amount of hydroperoxide is required to initiate and maintain the freeradical cyclooxygenase reaction. although large amounts of the peroxide inactivate the enzyme. When radical trapping anti-oxidant agents such as phenol are present, the radical intermediates decrease to lower levels which do not permit the reaction to continue. Therefore, the quantity of hydroperoxide present within the cell determines whether cyclooxygenase is activated or inactivated. It also modifies the inhibitory efficiency of cyclooxygenase inhibitors (13). For example, low concentrations of phenol, which are normally stimulatory. when combined with glutathione peroxidase result in potent synergistic inhibition. The inhibitory potency of phenolic non-steroidal anti-inflammatory drugs (NSAIDs) such as phenylbutazone and MK 447 are enhanced by reducing the peroxide, whereas the effect of non-antioxidant type drugs are not affected. Harada et al. (14) reported that MK 447 inhibited $P G E_{2}$ formation in vivo in the carrageenininduced pleurisy model and suggested that the anti-inflammatory effects of that compound was due to the inhibition of $\mathrm{PGE}_{2}$ synthesis. Although phenol and MK 447 have dual effects on PG biosynthesis in vitro, the anti-inflammatory effect of such compounds may be due to inhibition rather than stimulation of PG biosynthesis, as NSAIDs generally do. The present study has demonstrated the anti-edematous effects of phenolic dental medicaments which have either dualstimulatory and inhibitory effects or solely inhibitory effects on PG biosynthesis in vitro. Indomethacin also effectively inhibited inflammatory responses to croton oil in the present assay system. It has been demonstrated that the levels of PGE and PGF increased in mouse ears inflamed by phorbol ester, a component of croton oil, and anti- inflammatory drugs inhibited both edema and $P G$ formation (15). This evidence suggests the involvement of $P G s$ as mediators of inflammation in this model and the possible involvement of the inhibition of $\mathrm{PG}$ biosynthesis with phenolic dental medicaments in the mechanism for the anti-inflammatory effect of these compounds.

Phenolic medical preparations used in the treatment of pulpitis and as disinfectants for dentin contain high concentrations of phenolic compounds such as eugenol, guaiacol and phenol. Considering the amphiphilic property of these compounds and the reversible interaction with tissue protein and thus the capacity to penetrate into tissue. it is assumed that the tissue concentration of the compounds, when actually applied. could reach high enough levels to produce the pharmacological effects. Thus the phenolic compounds may produce diverse effects depending on their concentration in the tissue, non-specific denaturation of tissue. and specific inhibition of $P G$ biosynthesis. resulting in the analgesic and anti-inflammatory effects.

Acknowledgments: This work was supported in part by Grants-in Aid for Co-operative Reserch, No. A-59370043 and A-61304053, and a Grant-in Aid for Encouragement of Young Scientists. No. 56770897, from the Ministry of Education. Science and Culture, Japan.

\section{References}

1 Vane, J.: The evoluation of non-steroidal antiinflammatory drugs and their mechanisms of action. Drugs 33, 18-27 (1987)

2 Lindgren, J.A., Claesson, H.-E. and Hammarstoröm, S: Inhibition of prostaglandin synthesis in mouse $3 \mathrm{~T} 3$ fibroblasts and human platelets by substituted phenols. Prostaglandins 13, 1093-1102 (1977)

3 Dewhirst, F.E.: Structure-activity relationships for inhibition of prostaglandin cyclooxygenase by phenolic compounds. Prostaglandins 20, 209222 (1980)

4 Hirafuji, M.: Inhibition of prostaglandin $\mathrm{I}_{2}$ biosynthesis in rat dental pulp by phenolic dental medicaments. Japan. J. Pharmracol. 36, 544$546(1984)$

5 Anamura, S., Dohi, T., Shirakawa, M., Okamoto, H. and Tsujimoto, A.: Effects of phenolic dental medicaments on prostaglandin synthesis by 
microsomes of bovine tooth pulp and rabbit kidney medulla. Arch. Oral Biol. 33, 555-560 (1988)

6 Van Arman, C.G.: Anti-inflammatory drugs. Clin. Pharm. Ther. 16, 900-904 (1974)

7 Khuel, F.A., Jr., Humes, J.L., Egan, R.W., Ham, E.A., Beveridge, G.C. and Van Arman, C.G.: Role of prostaglandin endoperoxide $P G_{2}$ in inflammatory processes. Nature 265. 170-173 (1977)

8 Azuma, Y., Ozawa, N., Ueda, Y. and Takagi, N.: Pharmacological studies on the anti-inflammatory action of phenolic compounds. J. Dent. Res. 65, 53-56 (1986)

9 Payne, T.G., Dewald, B., Siegl, H., Gubler, H.U., Ott, $H$. and Baggiolini, M.: Radical scavenging and stimulation of prostaglandin synthesis not anti-inflammatory? Nature 296, 160-162 (1982)

10 Markey, C.M., Alward, A.r Weller, P.E. and Marnett, L.J.: Quantitative studies of hydroperoxide reduction by prostaglandin $H$ synthase. Reducing substrate specificity and the relationship of peroxidase to cyclooxygenase activities.
J. Biol. Chem. 262, 6266-6279 (1987)

11 Harada, Y., Tanaka, K. and Katori, M.: Dual effects of a basic anti-inflammatory agent, 2aminomethyl-4-t-butyl-6-iodophenol hydrochloride (MK-447), on biosynthesis of prostaglandin endoperoxides. Japan. J. Pharmacol. 30, 549-557 (1980)

12 Lands, W.E.M.: Actions of anti-inflammatory drugs. Trends Pharmacol. Sci. 2, 78-80 (1981)

13 Lands, W.E.M. and Hanel, A.M.: Phenolic anticylcooxygenase agents in anti-inflammatory and analgesic therapy. Prostaglandins 24, 271-277 (1982)

14 Harada, Y., Tanaka, K., Yamashita, K., Ishibashi, M., Miyazaki, H. and Katori, M.: The antiinflammatory mechanism of MK-447 in rat carrageenin-induced pleurisy. Prostaglandins 26. 79-90 (1983)

15 Carlson, R.P., O'Neill-Davis, L., Chang, J. and Lewis, A.J.: Modulation of mouse ear edema by cyclooxygenase and lipoxygenase inhibitors and other pharmacologic agents. Agents Actions 17 . 197-204 (1985) 